\section{Breath hold techniques during volumetric modulated arc therapy in breast cancer patients}

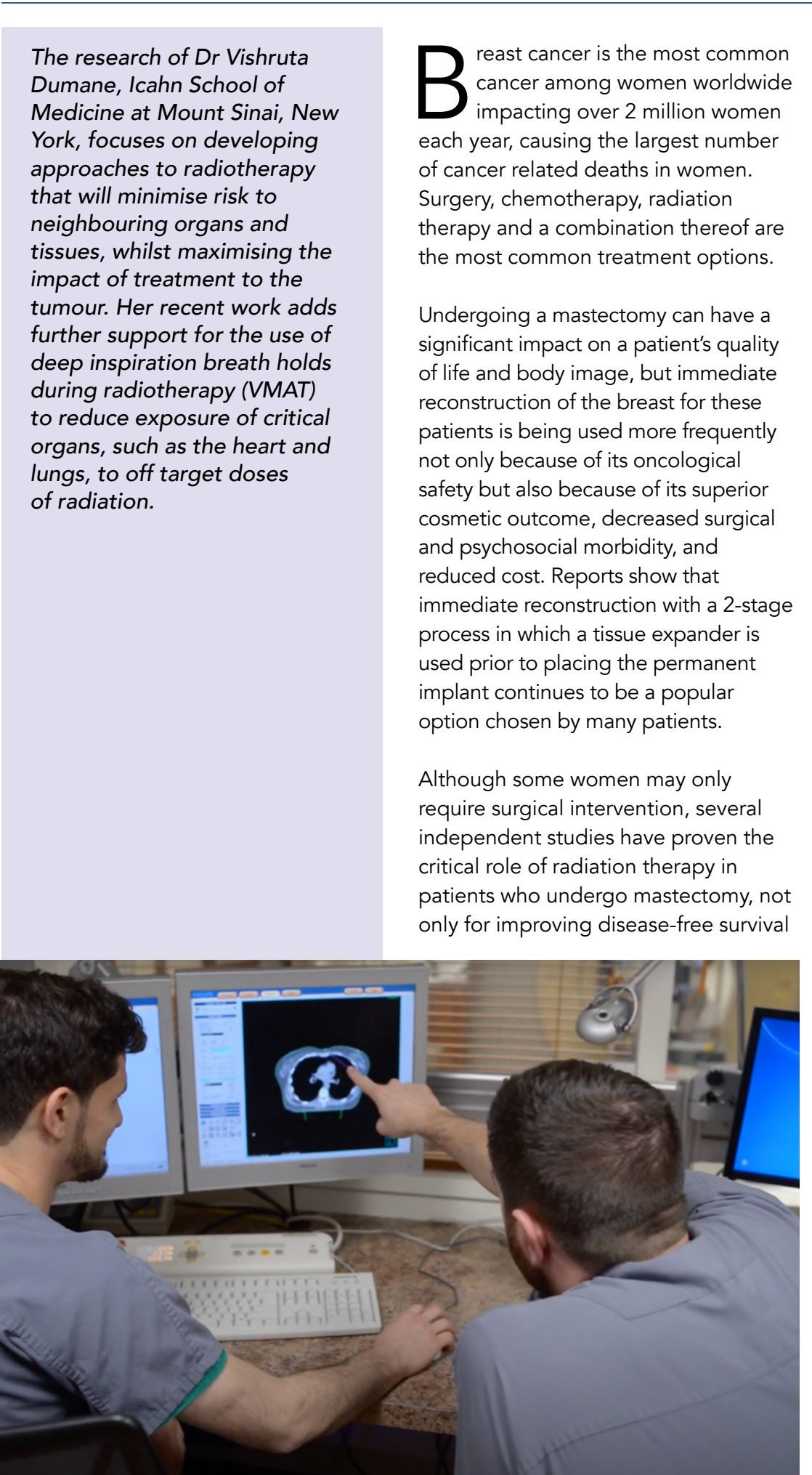

but also for reducing mortality rates from breast cancer, especially when the disease is advanced having spread to the nearby lymph nodes.

\section{RADIOTHERAPY}

OLLOWING MASTECTOMY

Traditionally, 3D conformal radiation

(a)

are shaped to adequately cover the

tumour while minimising the impact

of exposure to neighbouring critical

organs such as the heart and lungs. The

disadvantage of this approach, howeve,

is that it is difficult to achieve adequate

balance between these two goals

when there is a significant anatomica

challenge (e.g. the presence of a

tissue expander or a permanent breast

implant). In such situations, traditiona

techniques place the heart, lungs,

contralateral breast, as well as the rest of

the normal tissue at a risk of unnecessay

high dose exposure, which should

be minimised. Additionally, the need

which postentilly carn canchouses,

which potentially carry cancerous cells,

egion required to be targeted with

adiation, increasing the complexity

of treatment planning. The nodal

regions include the internal mammany

nodes (IMNs), axillary nodes and the

supraclavicular nodes. The IMNs form

chain from the inner medial portion of

the breast tissue by the sternum, along

the collarbone, to the armpit where

they join in with the axillary lymph

nodes. The supraclavicular lymph nodes

are above the collar bone and toward

the hollow of the neck. In the presence

of a tissue expander or a permanent

implant, it is even more challenging

to target the IMNs adequately with

radiation without significantly exposing

tissue to high doses.

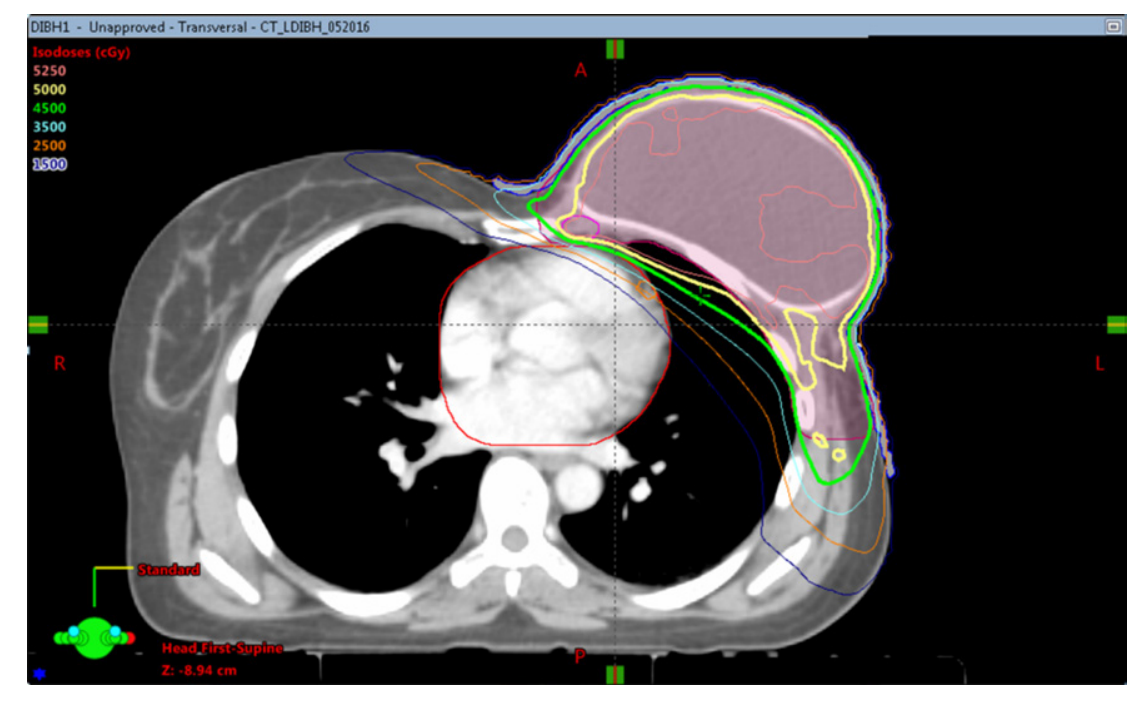

Dose distribution in the axial plane for a $\mathrm{DIBH}$ plan. As the
is pulled away from the chestwall as shown in this image.

One technique used to try and minimise the exposure of organs to unnecessary radiation in general is deep inspiration breath hold (DIBH).

It is this potential radiation exposure and there is a caveat, which is an increase damage that DrVishruta Dumane at the in exposure of unnecessary low dose Icahn School of Medicine at Mount Sinai, of radiation. This happens due to the New York, is trying to develop techniques continuous rotation of the machine and to limit. By utilising more advanced hence an increase in the use of machine treatment planning and delivery

techniques such as volumetric modulated arc therapy (NMAT), Dr Vishruta Dumane's work shows that these challenges faced in reducing high dose regions to critical organs and surounding normal tissue can be conform the opposed to thatlonal limited parameters and machine angles for deliver. VMAT is a technique that delivers radiation as the machine continuously rotates around the patient meaning that radiation delivery can be dynamically shaped and controlled, allowing many degrees of freedom to cover the tumour while optimally sparing critical organs. It also requires fewer monitor units compared to techniques with similar aims such as intensity modulated radiation therapy (MRT). This helps reduce the time required to treat the patient without compromising delivered dose of radiation.

Despite the advantages offered by VMAT in reducing high dose exposu angles of the radiation beam directed towards the patient. HOLD YOUR BREATH One technique used to try and minimise the exposure of organs to unnecessary breath hold (DIBH) DIBH is a tection tilised in many clinics with traditional 3D conformal radiation therapy to treat breast cancer. In this technique, the

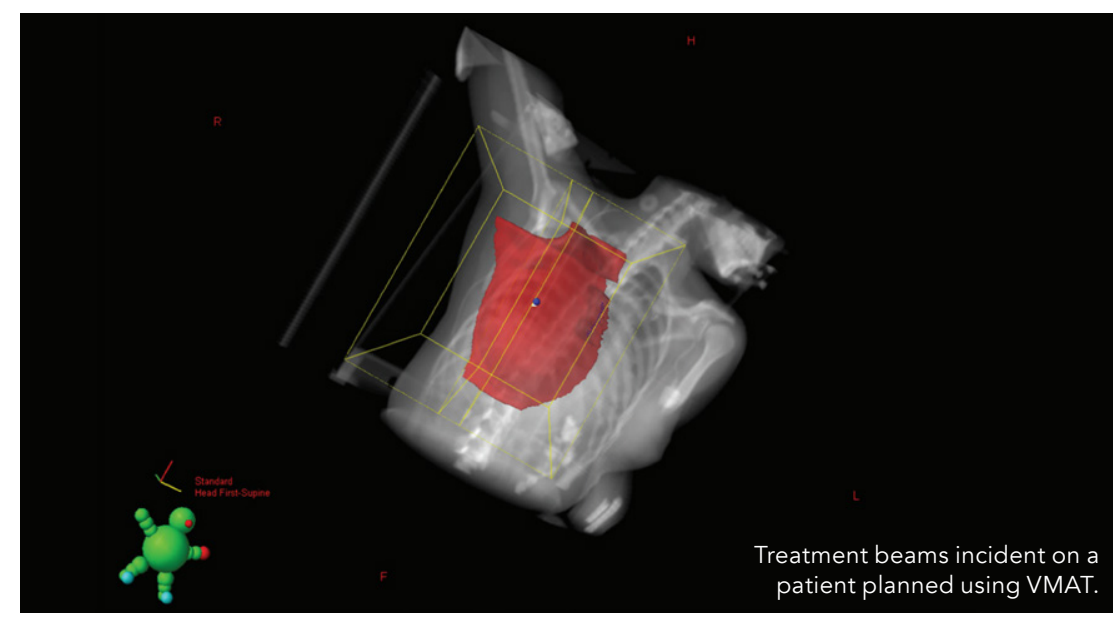


Interestingly, treatment machines used to deliver radiotherapy can be special sensors placed on the patient that tell the machine if the patient is not breathing enough that would help separate critical organs from the treatment area. This would automatically terminate the radiation beam, turning it back on only if the patient is able to breathe in adequately as expected. This arrangement manages to spare the critical organs and avoid excessive dose to normal tissue.

DEEP BREATH HOLDS REDUCE RADIATION EXPOSURE The main findings of Dr Dumane's study were that DIBH during VMAT did reduce the volume of the heart lungs and contralateral breast or radiation while also reducing the average radiation exposed to the heart. Given that there is a significant link between radiation dose that the heart is exposed to, and cardiac events later in life, this has important implications for patients with breast cancer undergoing radiotherapy.

In addition to reducing exposure to the heart, DIBH also decreased the level of radiation to the lungs, reducing the risk of radiation-induced inflammation
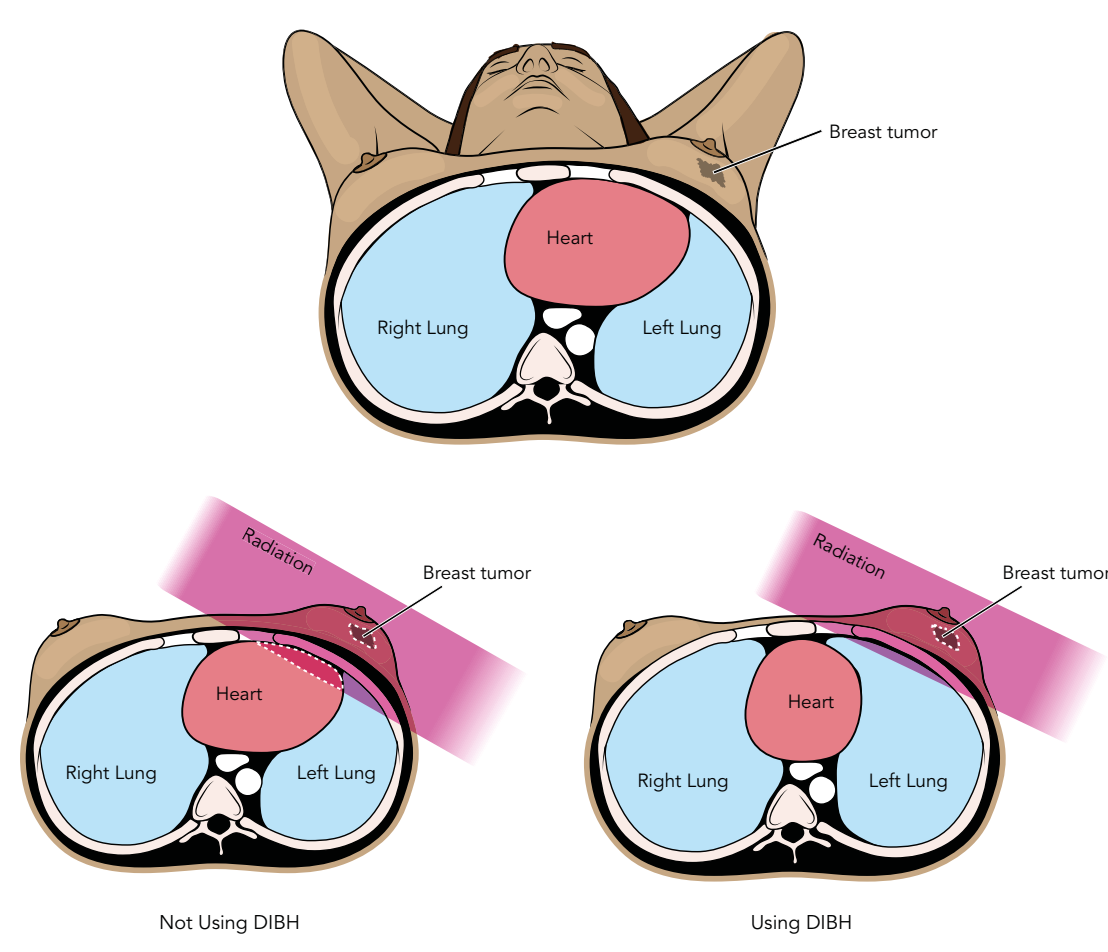

Graphical illustration of the benefit of DIBH. As the patient takes a deep breath, the heart is pull
away from the chestwall and away from the path of the radiation beam improving its sparing

Finally, the team were also able to show that DIBH reduced the amount of the contralateral breast exposed to radiation, reducing the risk of future incidence of secondary cancer to the contralateral breast.

Although DIBH as a technique is not novel and has already been

implemented across many treatment

shows

Using a combination of VMAT and DIBH reduced radiation to the heart and lungs by $30 \%$ and $11 \%$, respectively, and to the contralateral breast/implant by $18 \%$.

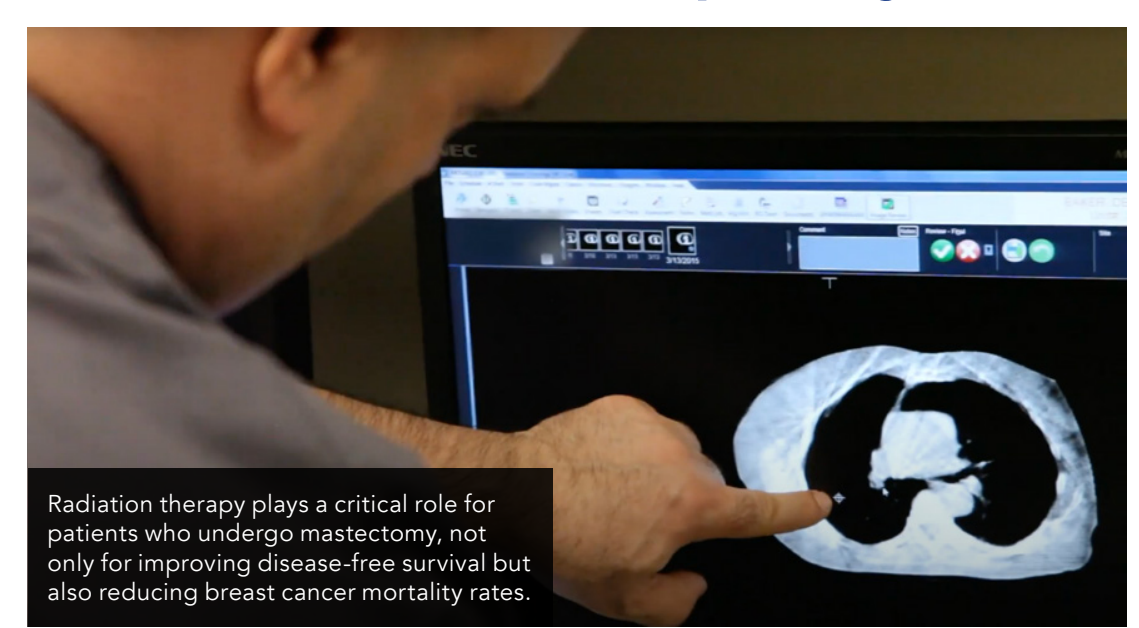

its application in patients receiving VMAT for complex anatomies due to the presence of tissue expanders or permanent implants requiring regional nodal irradiation, furthering knowledge and understanding to the ways in which patients with breast cancer undergo treatment. DIBH when dosicis dosimentic impact with respect to overall ben fose which is of immense consequences - especially since the exposure in this cohort of pariese relatively understudied and unknown.

Dr Dumane concludes that DIBH should patients with implant reconstructions also requiring regional nodal radiation are being treated with VMAT due to complex anatomies. Using a combination of VMAT and DIBH reduced radiation to the heart and lungs by $30 \%$ and $11 \%$, respectively, and to the contralateral breast/implan by $18 \%$. This suggests that using a breath hold is beneficial and should be factored into treatment planning for this patient group, especially as there is Imited to no risk associated with it, an for a treatment centre with machines configured to use this technology should require no addition

Dr Vishruta Ajitkumar Dumane

E: Vishruta.dumane@mountsinai.org T: +1 2125235330 W: https://vishrutadumane.academia.edu/

\section{Research Objectives}

Dr Dumane investigates the development of treatment planning approaches that are designed to cover the target volumes while minimising dose to critical organs, developing knowledge-based models for automated treatment planning for various treatment sites including radiosurgery (SRS) and prostate cancer.

\section{Detail}

Department of Radiation Oncology

Mount Sinai West 100010 th Avenue

New York NY, 10019

Bio

DrVishruta Ajitkumar Dumane is a Medical Physicist and Assistant Professor of Radiation Oncology at Icahn Schoo of Medicine at Mount Sinai. She joined the Mount Sinai Sloan-Kentering Cancer Center in 2005. She has aumoria a book chapter on intensity modulated radiation therapy (IMRT) for breast cancer as well as several publications on the topic of breast treatment planning including reducing dose to organs in the supraclavicular region, use of DIBH to reduce low dose, combining 3D and VMAT to improve regional nodal irradiation and optimising treatment positioning to achieve better heart sparing for a left-sided breast cancer case, the latter of which was awarded the cology, 2016

Funding

This research is supported by the MSK Cancer Center Support Grant/Core: Grant (P30 CA008748).

\section{Collaborators} Powell, M.D., Ying Zhou, M.S., Linda Hong, Ph.D.

The study design and analyses was performed by the corresponding author. All patients were treated at (New York, NY)

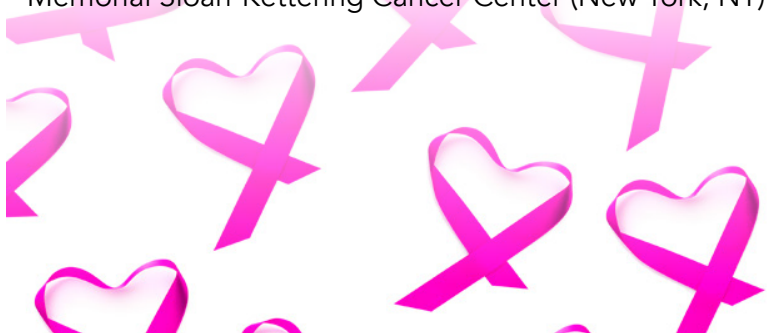

\section{References}

Dumane, V.A, Saksornchai, K., Zhou, Y., Hong, L., Powell, S. \& Ho, A.Y. (2018). Reduction in low-dose to normal tissue with the addition of deep inspiration breath hold (DIBH) to volumetric modulated arc therapy (VMAT) in breast cancer patients with implant reconstruction receiving regional 13(187), https://do org/10.1186/s13014-018-1132-9.

\section{Personal Response}

What other techniques can be used to limit the exposure of surrounding tissues and organs to high

IU Techniques to limit high doses of radiation to the surrounding tissue are field-in-field intensity modulated to reduce volumes of high doses throughout the target: however these are typically restricted to 2 radiation beams. Multibeam intensity-modulated radiation therapy (MB-IMRT) can further reduce high dose regions to surrounding tissue since it uses many radiation beams from different angles/approaches. Helical tomotherapy $(\mathrm{HT})$ is another way of delivering MB-IMRT where the machine resembles a CT scanner continuously rotating 政 techniques mentioned use photons for treathent. Th other option is to use proton therapy

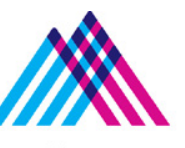

Icahn

School of

Medicine at

Mount

Sinai

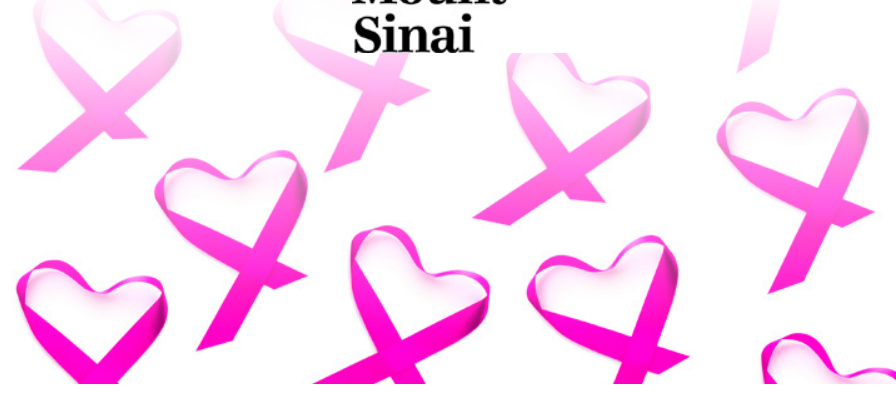

